\title{
Gravitational waves from phase transitions at the electroweak scale and beyond
}

\author{
Christophe Grojean ${ }^{1,2, *}$ and Géraldine Servant ${ }^{1,2, \dagger}$ \\ ${ }^{1}$ CERN, Theory Division, CH-1211 Geneva 23, Switzerland \\ ${ }^{2}$ Service de Physique Théorique, CEA Saclay, F91191 Gif-sur-Yvette, France
}

(Received 13 September 2006; published 12 February 2007)

\begin{abstract}
If there was a first-order phase transition in the early universe, there should be an associated stochastic background of gravitational waves. In this paper, we point out that the characteristic frequency of the spectrum due to phase transitions which took place in the temperature range $100 \mathrm{GeV}-10^{7} \mathrm{GeV}$ is precisely in the window that will be probed by the second generation of space-based interferometers such as the big bang observer (BBO). Taking into account the astrophysical foreground, we determine the type of phase transitions which could be detected either at LISA, LIGO or BBO, in terms of the amount of supercooling and the duration of the phase transition that are needed. Those two quantities can be calculated for any given effective scalar potential describing the phase transition. In particular, the new models of electroweak symmetry-breaking which have been proposed in the last few years typically have a different Higgs potential from the standard model. They could lead to a gravitational wave signature in the milli-Hertz frequency, which is precisely the peak sensitivity of LISA. We also show that the signal coming from phase transitions taking place at $T \sim 1-100 \mathrm{TeV}$ could entirely screen the relic gravitational wave signal expected from standard inflationary models.
\end{abstract}

DOI: 10.1103/PhysRevD.75.043507

PACS numbers: 98.80.Cq, 04.30.Db, 11.10.Wx

\section{INTRODUCTION}

Direct detection of gravitational radiation will hopefully soon become a reality, with the operation of the first generation of interferometers such as (kilometer-scale, ground-based) LIGO [1] and VIRGO [2] and (millionkilometer-scale, space-based) LISA [3]. Those instruments will allow us to probe gravitational waves produced by astrophysical objects at relatively low red-shift (black hole binaries, neutron star binaries, white dwarf binaries, supernovae, pulsars...) In addition, gravitational waves (GW) can provide information about particle physics at unexplored high energies. The weakness of the interaction with matter is a major obstacle for detection of gravitational waves but it also has the virtue that the information they carry about the state of the universe at the moment of their production has been unaltered. They are precious information on the very mechanism that produced them. GW can be produced by core collapse of supernovae, first-order phase transitions, vibration of cosmic strings, preheating, dynamics of extra dimensions, etc.

Among those well-motivated but hypothetical cosmological sources of $\mathrm{GW}$, there is at least one that we are convinced exists: the GW produced during inflation. This signal is expected to be very tiny. Quantum fluctuations in the inflaton field during inflation leaves behind a residue in density perturbations observed in the cosmic microwave background (CMB). They also lead to a background of GW whose properties couple with those of density fluctuations. As the CMB anisotropies are affected by GW, the WMAP constraint on the energy scale of inflation fixes a bound on

\footnotetext{
*Electronic address: christophe.grojean@cern.ch

†Electronic address: geraldine.servant@cern.ch
}

the size of the GW signal due to inflation $\Omega_{\mathrm{GW}} h^{2} \lesssim$ $10^{-15}-10^{-14}$ [4]. This is several orders of magnitude below the best sensitivity of the first-generation of interferometers. However, attempts to detect this relic primordial background are very strongly motivated. This is a main goal of the second generation of space interferometers, in particular, the big bang observer (BBO) [5], the follow-on mission to LISA, which would become a reality within 20 or 30 years (by comparison, LISA, if funded, should be operational by 2014).

The present work focusses instead on the detectability of $\mathrm{GW}$ from first-order phase transitions. The corresponding relic GW background encodes useful information on these major symmetry-breaking events which took place in the early universe. In contrast with the inflationary spectrum, the spectrum is not flat, with a characteristic peak related to the temperature at which the phase transition (PT) took place. This signal can actually be higher by several orders of magnitude than the signal expected from inflation and in some cases can entirely screen it. One symmetry-breaking event which for sure took place in the early universe is electroweak (EW) symmetry breaking. What we do not know yet is whether it was a first-order phase transition, in which case it proceeded through nucleation of bubbles resulting in a large departure from thermal equilibrium. Bubble collision and associated motions in the primordial plasma are sources of gravitational waves. The characteristic frequency of the signal is close to the Hubble frequency at the time of the transition $H\left(T_{\mathrm{EW}}\right) \sim 10^{-14} \mathrm{GeV}$. Once redshifted to today, this corresponds to $\mathrm{mHz}$ frequencies, which is precisely the frequency band that LISA is sensitive to. It is therefore very exciting that LISA could help providing information on the EW scale, in particular, on the nature of the EWPT. 
The GW spectrum resulting from first-order PT was computed in the early nineties [6-9] but this topic has not received much subsequent attention, as it was found out that there is no first-order EWPT in the standard model given the experimental bound on the Higgs mass [10]. It was realized ten years after the original calculation of [69] that turbulence in the plasma could be a significant source of GW in addition to bubble collisions [11,12]. Subsequently, the authors of [13] studied the GW signal due to a first-order EWPT in the minimal supersymmetric standard model (MSSM) and its NMSSM extension. Finally, Nicolis [14] did a model-independent analysis for the detectability of GW with LISA.

We believe that it is time to revisit this question for two reasons: The nature of the EWPT will start to be probed experimentally at the LHC. Indeed, it depends essentially on the Higgs sector of the theory or any alternative dynamics for EW symmetry breaking. In the last few years, new models of EW symmetry breaking have been suggested (little Higgs, gauge-Higgs unification, composite Higgs, Higgsless models...) and the nature (smooth crossover or first-order) of the EWPT in these new frameworks remains unknown. Second, the technology for gravitational wave detectors has made advances [15] and we think it is timely to redo a model-independent analysis not only for LISA but also other devices.

LIGO is sensitive to much higher frequencies (from a few $\mathrm{Hz}$ to a few hundreds of $\mathrm{Hz}$ ) thus it is in principle sensitive to phase transitions which took place at much earlier epochs. For instance, we will show that if there was a very strong first-order PT at temperatures of order $10^{7} \mathrm{GeV}$, the ultimate stage of LIGO (LIGO-III, correlated) could detect the corresponding peak (as already pointed out in [6]). The second generation of interferometers will be able to say much about the possible existence of early universe first-order phase transitions. Indeed, BBO will be sensitive to signals from PT which would have taken place in the temperature range $T \sim$ $100 \mathrm{GeV}-10^{7} \mathrm{GeV}$, even if not necessarily exceptionally strong.

In this paper, we start with some generalities on stochastic GW backgrounds including the astrophysical background. We also recap what would be the observable redshifted signal we would observe after the GW have propagated forward from the phase transition until today. Section III reviews the key formulas used in the theoretical predictions of the GW spectrum due to first-order phase transitions. There is nothing new in this part. However, this formalism had so far only been exploited to study the detectability at LISA of GW due to a first-order electroweak phase transition. In Sec. IV, we apply it to any other phase transitions taking place at higher temperatures and compare them with the sensitivities of not only LISA but also LIGO and BBO. Predictions can be presented in a model-independent way as a function of two quantities, namely $\alpha$ ( $\sim$ latent heat $)$ and $\beta^{-1}$ ( $\sim$ duration of the phase transition), which can be computed for any given effective scalar potential describing the transition. For each temperature, we identify which values of $\alpha$ and $\beta$ lead to an observable signal. Particle model builders can then test their favourite scalar potential by computing its corresponding values of $\alpha$ and $\beta$ and see whether it can give rise to a detectable GW signal. We comment on some specific examples of particle physics models.

\section{ASTROPHYSICAL VERSUS COSMOLOGICAL GW BACKGROUND}

Stochastic backgrounds are random gravitational waves arising from the incoherent superposition of a large number of independent, uncorrelated sources that cannot be resolved individually. They are discussed in terms of their contribution to the universe's energy density, over some frequency band:

$$
\Omega_{\mathrm{GW}}(f)=\frac{1}{\rho_{\text {crit }}} \frac{d \rho_{\mathrm{GW}}}{d \ln f} .
$$

By their very nature, stochastic GW are indistinguishable from the detector noise. Ground-based detectors look for them by coordinated measurements (comparing outputs of multiple detectors to find sources of correlated noise) while LISA can extract the instrumental noise power by combining the signals from its three spacecrafts. For technical aspects related to the detection of a gravitational wave stochastic background, see Ref. [16].

Searching for GW waves of cosmological origin is an ambitious goal. There is a huge foreground due to astrophysical sources which in principle makes detection impractical. Once the signals from every merging neutron star and stellar mass black holes have been identified and substracted, the primary sources of foreground signals are galactic and extragalactic binaries. The galactic background produced by binary stars in the Milky Way is many times larger in amplitude than both the extragalactic foreground and LISA's design sensitivity. However, it can be substracted because of its anisotropy, being mostly concentrated in the galactic plane. Irreducible background comes from extragalactic binary stars and is dominated by emission from white dwarves (WD) pairs. The corresponding GW spectrum was estimated in Ref. [17] where limits are placed on the minimum and maximum expected background signals. Ref. [17] points out that at frequencies $f \lesssim 50 \mathrm{mHz}$, there will be too many individual WD-WD sources contributing in each resolution element to be completely resolved and substracted source by source by missions with plausible lifetimes. However, much of the flux comes from relatively nearby sources, and the WD-WD numbers drop rapidly above $50 \mathrm{mHz}$. Thus it may be possible for future missions more sensitive than LISA to substract this background at high frequencies [17]. In our 
figures, we plot this background coming from unresolved compact white dwarf binaries assuming that it can be removed at frequencies above $50 \mathrm{mHz}$. At higher frequencies, the dominant foreground $\mathrm{GW}$ sources are inspiralling neutron star-neutron star, neutron star-black hole and black hole-black hole binaries. These have to be individually identified and substracted. This problem is discussed in [18], and in our BBO detectability analysis we optimistically assume that this foreground can be substracted.

The GW background due to early universe events is stochastic as the signal comes from the superposition of incoherent sources originating from a huge number of different horizon volumes. For instance, the size of the horizon at the time of the electroweak phase transition was much smaller than today $\left(10^{-14} \mathrm{GeV}\right)^{-1}$, corresponding to a tiny fraction of degree on the sky today. Gravity waves produced at the temperature $T_{*}$ with a characteristic frequency $f_{*}$ propagate until today without interacting. Their energy density redshifts as $a^{-4}$ and their frequency as $a^{-1}$ (we assume a radiation-dominated era). The characteristic frequency of the signal we observe today is

$$
\begin{aligned}
f & =f_{*} \frac{a_{*}}{a_{0}}=f_{*}\left(\frac{g_{s 0}}{g_{s *}}\right)^{1 / 3} \frac{T_{0}}{T_{*}} \\
& \approx 6 \times 10^{-3} \mathrm{mHz}\left(\frac{g_{*}}{100}\right)^{1 / 6} \frac{T_{*}}{100 \mathrm{GeV}} \frac{f_{*}}{H_{*}},
\end{aligned}
$$

where $H_{*}$ and $g_{*}$ are, respectively, the Hubble frequency and the number of relativistic at the temperature $T_{*}$. The remarkable fact is that for $T \sim 100 \mathrm{GeV}$ and $f_{*} / H_{*} \sim 10^{2}$, (as expected for weak scale processes as will be explained below), the peak frequency of the GW spectrum is in the milli-Hertz, just in the band of LISA.

\section{GW FROM FIRST-ORDER PHASE TRANSITIONS}

\section{A. A two parameter problem}

Phase transitions are commonly described by the effective potential of the scalar field (either elementary or composite) responsible for the dynamics. First-order phase transitions are triggered if there exists a temperature at which a barrier separates two degenerate minima. Then, the phase transition proceeds via nucleation of bubbles which occurs through quantum tunneling and thermal fluctuations. As a bubble expands, part of the liberated latent heat raises the plasma temperature while the other part is converted into kinetic energy of the bubble wall and bulk motions of the fluid. Because of its spherical symmetry, a single expanding bubble produces no gravity waves. Only after bubble collisions destroy the spherical symmetry is gravitational radiation emitted. High velocities and large energy densities provide the necessary conditions for producing gravitational radiation. There are two sources of gravitational waves: the actual collision of bubbles and the turbulence in the plasma due to bubble motion. The result- ing spectrum of gravitational waves has been studied in details in [6-9,11-14]. The turbulence spectrum was recently revisited in Ref. [19]. Reexamination of the bubble collision spectrum is underway [20].

We are working under the assumption that bubble expansion proceeds via detonation [21], which is justified for strong first-order phase transitions only. Remarkably, in this regime, GW predictions only depend on the grossest features of the bubble collisions.

The first important parameter is the rate of variation of the bubble nucleation rate, called $\beta$. This quantity fixes the characteristic scale in the problem, the size of bubbles at the time of the collision, and therefore the characteristic frequency $f_{*}$ of the GW spectrum. The duration of the phase transition is given by $\beta^{-1}$ and the size of bubbles is typically $R_{b} \sim v_{b} \beta^{-1}$ where $v_{b}$ is the velocity of the bubble wall. The initial size of the bubble at the time of nucleation (of the order of $T^{-1}$ ) is negligible compared to $\beta^{-1}$ which is of the order of the horizon size. The second crucial parameter characterizing the spectrum of gravitational waves is $\alpha=\epsilon / \rho_{\text {rad }}$, the ratio of the latent heat liberated at the phase transition $(\epsilon=$ latent heat) to the energy density in the high energy phase, commonly being radiation energy density. $\epsilon$ is not necessarily vacuum energy, see for instance [22]. $\alpha$ and $\beta$ are evaluated at the nucleation temperature and determine entirely the $\mathrm{GW}$ spectrum.

The parameters $\alpha$ and $\beta$ can be computed once we know the effective action for nucleating bubbles ("critical bubbles") which can be computed for any scalar potential describing the phase transition, though the numerical computation is rather delicate. The critical bubbles extremize the 3D Euclidean action

$$
S_{3}=\int 4 \pi r^{2} d r\left(\frac{1}{2}\left(\frac{d \phi_{b}}{d r}\right)^{2}+V\left(\phi_{b}, T\right)\right) .
$$

It is equivalent to the differential system

$$
\begin{gathered}
\frac{d^{2} \phi_{b}}{d r^{2}}+\frac{2}{r} \frac{d \phi_{b}}{d r}-\frac{\partial V}{\partial \phi_{b}}=0 \quad \text { with }\left.\frac{d \phi_{b}}{d r}\right|_{r=0}=0 \quad \text { and } \\
\left.\phi_{b}\right|_{r=\infty}=0,
\end{gathered}
$$

that can be solved using an overshooting-undershooting method. The phase transition completes when the probability for the nucleation of 1 bubble per 1 horizon volume and horizon time is of order 1 , which guarantees that bubbles percolate even if the universe is in inflation. This condition translates into ( $T_{\star}$ is the temperature at time $t_{\star}$ when the phase transition completes)

$$
S_{3}\left(T_{\star}\right) / T_{\star} \sim-4 \ln \left(T_{*} / m_{\mathrm{Pl}}\right) .
$$

The time variation of the rate of bubble nucleation, $\Gamma(t)=$ $A(t) e^{-S(t)}$, is mostly controlled by $S(t) \approx S_{3} / T$. Thus

$$
\beta \equiv-\left.\left.\frac{d S}{d t}\right|_{t_{*}} \approx \frac{1}{\Gamma} \frac{d \Gamma}{d t}\right|_{t_{*}},
$$


and, using the adiabaticity of the expansion of the universe, $d T / d t=-T H$ ( $H$ is the expansion rate of the universe), we obtain

$$
\frac{\beta}{H_{*}}=\left.T_{*} \frac{d S}{d T}\right|_{T_{*}}=\left.T_{*} \frac{d}{d T}\left(\frac{S_{3}}{T}\right)\right|_{T_{*}} .
$$

The latent heat is the sum of two contributions. The first one is the difference in free energies between the stable and metastable minima (which vanishes at $T_{c}$ ) while the second one comes from the entropy variation $\Delta s$ (which is non zero at $T_{c}$ in a first-order PT). This leads to the following formula for $\epsilon$ :

$$
\epsilon=-\Delta V-T \Delta s=(-\Delta V+T \partial V / \partial T)_{T_{*}}
$$

\section{B. Scaling expectations and GW spectrum}

The energy density in gravitational waves can be deduced from the quadrupole formula for the power of gravitational emission. Let $\kappa$ be the efficiency factor which quantifies the fraction of the vacuum energy which goes into kinetic energy of bulk motions of the fluid (as opposed to heating). In a radiation-dominated FRW universe, the redshifted value of the energy density evaluated today at the peak frequency is [9]:

$$
\begin{aligned}
\Omega_{\text {coll }} h^{2}\left(f_{\text {coll }}\right) \simeq & 1.1 \times 10^{-6} \kappa^{2}\left[\frac{H_{*}}{\beta}\right]^{2}\left[\frac{\alpha}{1+\alpha}\right]^{2} \\
& \times\left[\frac{v_{b}^{3}}{0.24+v_{b}^{3}}\right]\left[\frac{100}{g_{*}}\right]^{1 / 3},
\end{aligned}
$$

if the gravitational waves are produced by bubble collision ( $g_{\star}$ is the number of relativistic degrees of freedom at $T_{\star}$ ). The peak frequency of the collision spectrum is [9]

$$
f_{\text {coll }} \simeq 5.2 \times 10^{-3} \mathrm{mHz}\left[\frac{\beta}{H_{*}}\right]\left[\frac{T_{*}}{100 \mathrm{GeV}}\right]\left[\frac{g_{*}}{100}\right]^{1 / 6} .
$$

The scaling is slightly different in the case of GW from turbulence in the plasma (the analysis of turbulent motions of [11] was generalized in $[12,14])$ :

$$
\Omega_{\text {turb }} h^{2}\left(f_{\text {turb }}\right) \simeq 1.4 \times 10^{-4} u_{s}^{5} v_{b}^{2}\left[\frac{H_{*}}{\beta}\right]^{2}\left[\frac{100}{g_{*}}\right]^{1 / 3},
$$

with $[12,14]$

$$
f_{\text {turb }} \simeq 3.4 \times 10^{-3} \mathrm{mHz} \frac{u_{s}}{v_{b}}\left[\frac{\beta}{H_{*}}\right]\left[\frac{T_{*}}{100 \mathrm{GeV}}\right]\left[\frac{g_{*}}{100}\right]^{1 / 6} .
$$

These formulas follow from an analysis assuming an idealized simplified model of turbulence. The turbulent fluid velocities $u_{s}$ are smaller than the bubble expansion velocities, unless turbulence is extremely strong. As a result, according to (10) and (12), the peak for the turbulence spectrum will be shifted to lower frequencies. Note that the peak frequency of the collision signal does not depend on $\alpha$. Because $f_{\text {turb }} \approx f_{\text {coll }} \times\left(u_{s} / v_{b}\right)$ and $\left(u_{s} / v_{b}\right)$ is an increasing function of $\alpha$, as $\alpha$ increases, the collision peak gets hidden by the high-frequency tail of the turbulence signal. ${ }^{1}$

The signal from turbulence is more promising than the signal from bubble collision due to the different scaling with $\alpha$. We will use the following formulas for the velocities and the efficiency factor $[9,14,22]$ :

$$
v_{b}(\alpha)=\frac{1 / \sqrt{3}+\sqrt{\alpha^{2}+2 \alpha / 3}}{1+\alpha}, \quad u_{s}(\alpha) \simeq \sqrt{\frac{\kappa \alpha}{\frac{4}{3}+\kappa \alpha}}
$$

$$
\kappa(\alpha) \simeq \frac{1}{1+0.715 \alpha}\left[0.715 \alpha+\frac{4}{27} \sqrt{\frac{3 \alpha}{2}}\right] .
$$

We remind that Eqs. (13) and (14) are only valid if the bubbles propagate as relativistic detonations. This assumption allows us to express the final results in a modelindependent way, as a function of $\alpha$ and $\beta$ only. Note that the formula for $v_{b}$ neglects bubble friction due to interactions of the bubble wall with the particles in the plasma. In principle, $v_{b}=\epsilon / \eta$ where the friction $\eta$ depends on the width of the bubble wall which cannot be expressed in terms of $\alpha$ only [23]. The detonation assumption is reasonable for very strong phase transitions, but not for weaker ones, - bubbles can also propagate as subsonic deflagrations [21], in which case the radiation amplitude is much lower. Therefore, it makes no sense to take the $\alpha \rightarrow$ 0 limit in our formulas. Anyhow, it is clear that the signal is going to be detectable only for relatively large $\alpha$ in which case these formulas are applicable.

\section{OBSERVABILITY OF A GW SIGNAL FROM A PHASE TRANSITION}

The formulas (9) and (11) for $\Omega h^{2}$ show that the amplitude of the signal does not depend on the energy scale of the transition but only on the dimensionless parameters $\alpha$ and $H_{*} / \beta$, in other words on the shape of the scalar potential at the time of nucleation. Very roughly, taking $\beta / H_{*} \sim S_{3}\left(T_{*}\right) / T_{*} \sim \mathcal{O}(100)$, it is clear that we need $\alpha \sim$ $\mathcal{O}(1)$ if we want $\Omega h^{2} \gtrsim 10^{-10}$ (to see something at LISA). Our experience with possible values of $\alpha$ and $\beta / H_{*}$ is based on the studies of potentials describing the electroweak phase transition. The values of $\alpha$ and $\beta / H_{*}$ are related to the ratio $\phi\left(T_{*}\right) / T_{*}$, another quantity characterizing the strength of the phase transition, where $\phi$ is the

\footnotetext{
${ }^{1}$ Although the relation $f_{\text {turb }} \approx f_{\text {coll }} \times\left(u_{s} / v_{b}\right)$ is likely to be revised in Ref. [20].
} 
vacuum expectation value of the Higgs. For instance, in the MSSM, $\alpha$ is typically smaller than 0.1 while $\beta / H_{*}$ is larger than 1000 [13]. On the other hand, in the NMSSM, the authors of [13] found values of $\alpha \sim \mathcal{O}(1)$. Values of $\alpha$ larger than 1 correspond to a phase transition which is so strong that it is at the borderline of not being able to take place because the free energy of a critical bubble is too large: either the barrier separating the two minima is too large (the barrier even exists at zero temperature) or the distance in field space between the two minima of the potential is too big. The same applies if $\beta / H_{*}$ is smaller than $\mathcal{O}(100)$. This situation is encountered not only in the NMSSM but also in effective theories with large negative quartic couplings [24], as will be presented in [25]. Note that for a given scalar potential, $\alpha$ and $\beta / H_{*}$ are actually correlated as a large value of $\alpha$ will be associated with a small value of $\beta / H_{*}$. Indeed, $\alpha$ is proportional to the latent heat, which grows as $\Delta V$, the depth of the potential at the minimum, is getting bigger. On the other hand, $S_{3} / T$ (and thus $\beta / H_{*}$ ) typically scales like $1 / \sqrt{\Delta V}$.

Let us make a few comments concerning the situation where $\alpha \sim 1$. In usual phase transitions, this means that the vacuum energy is of the same order as the radiation energy density, therefore inflation starts before bubbles percolate.
However, if $\Gamma / H^{4} \sim \mathcal{O}(1)$ is satisfied, this guarantees that the phase transition can still complete. The number of efoldings of inflation is $\ln \left(V^{1 / 4} / T_{*}\right)$, which is typically less than 1 . However, if the transition is very slow, i.e. $\beta / H \lesssim$ 1 , one should take formulas (9) and (11) with caution as they are derived neglecting the expansion of the universe, which is not a good approximation if $\beta / H<1$.

Figure 1 shows some GW spectra illustrating the predictions for various temperatures with representative values of $\alpha=0.4,1$ and $\beta / H_{*}=100,800,3000$. Those plots also exhibit two examples of signals from inflation (and taken from [4]) for comparison. The scale of inflation is constrained by the CMB to be $E_{I} \lesssim 3.4 \times 10^{16} \mathrm{GeV}$ [4]. This fixes the largest signal we could expect from inflation as $\Omega_{\mathrm{GW}} h^{2} \propto E_{I}^{4}$. We also include the signal corresponding to $E_{I}=5 \times 10^{15} \mathrm{GeV}$ which could be observed at BBO.

We now want to characterize, in the $\left(\alpha, \beta / H_{*}\right)$ parameter space, phase transitions that leave a gravitational waves background obvervable either by LISA, BBO or LIGO.

In our analysis, we will use the formulas of the previous section as well as the fact that the spectrum $\Omega_{\text {coll }} h^{2}$ is expected to increase as $f^{2.8}$ while at high frequencies it drops off as $f^{-1.8}$ and that $\Omega_{\text {turb }} h^{2}$ increases as $f^{2}$ while at high frequencies it drops off as $f^{-7 / 2}$. This is already all
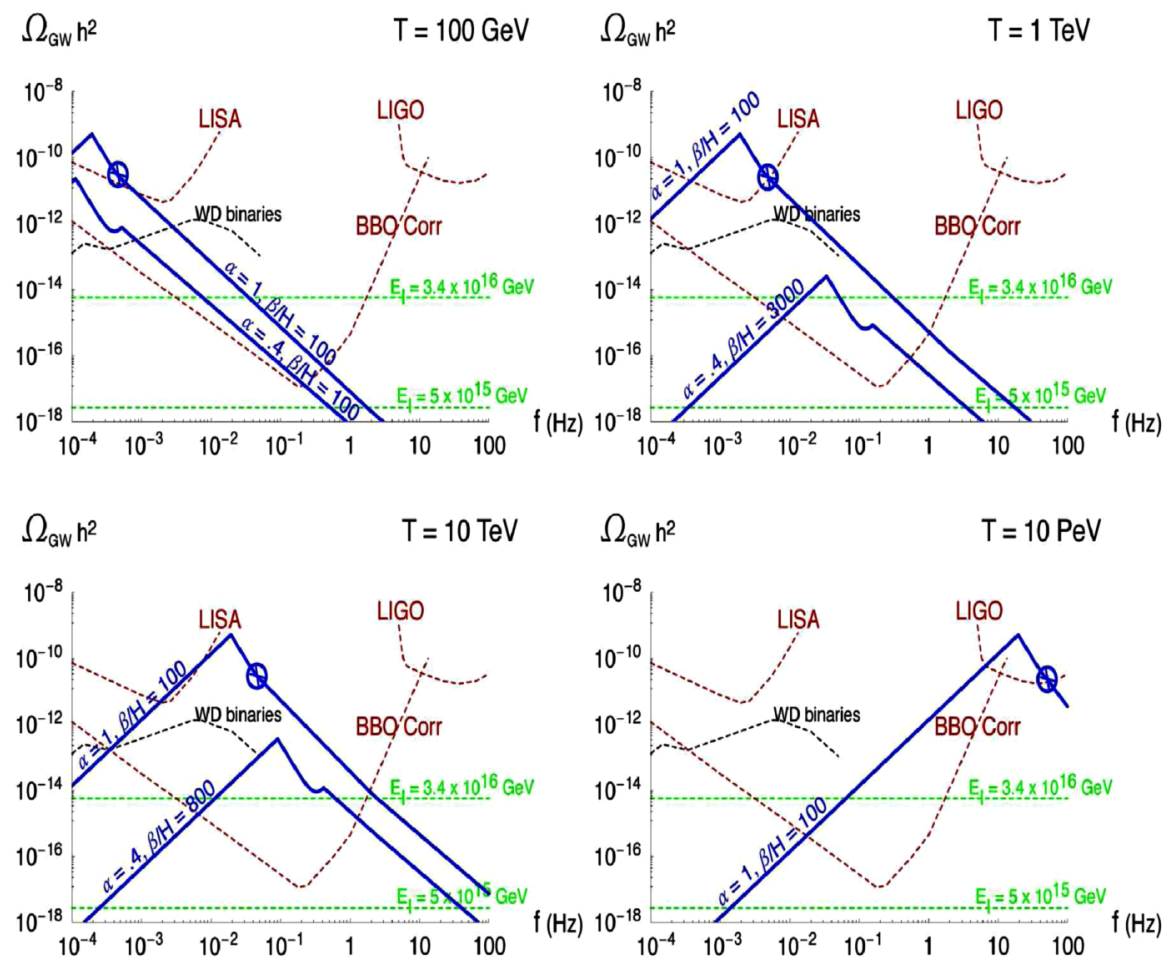

FIG. 1 (color online). Spectrum of gravitational waves expected from a first-order phase transition (solid blue line) for four temperatures and for some choices of $(\alpha, \beta / H)$ values. The dashed red lines are the (approximate) predicted sensitivities of LISA, BBO, LIGO-III. The horizontal dashed green lines are the gravitational spectra expected from inflation, for two scales of inflation, for comparison. The black dashed curve is the estimate for the irreducible foreground due to white dwarf binaries (from [17]). At large $\alpha$, only the peak from turbulence can be seen as well as a change of slope (shown as a circled cross) corresponding to the high-frequency tail of the bubble collision spectrum. For low $\alpha$, it is possible to see the collision peak as well. 
$\beta / \mathrm{H}$

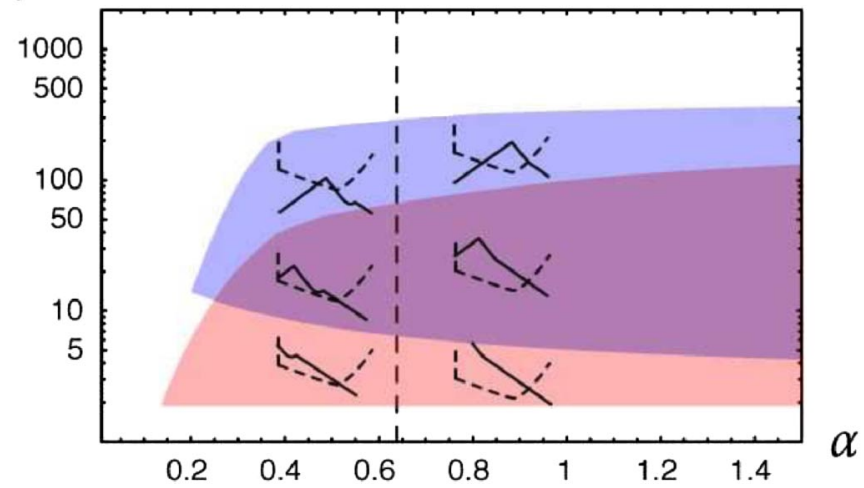

FIG. 2 (color online). Different configurations of the signal versus the instrument sensitivity to show the qualitative dependence on parameters. The upper blue region is where the turbulence peak is observable while the lower red one is the region where either the collision peak or the point of slope change is visible. Precise locations of these different regions depend on the experiment and the temperature of the transition as illustrated in Figs. 3-5.

summarized in the letter [14]. However, Ref. [14] focuses on the detectability at LISA of GW from a $T=100 \mathrm{GeV}$ phase transition, while we are now using this formalism to look in more details at the detectability of GW coming from any other 1 st order phase transitions at future interferometers. We repeat that we are working at the level of an-order-of-magnitude estimate. Magnetohydrodynamical effects could make the slope of the turbulence highfrequency tail smaller [14] and in any case, for a more precise analysis, the calculation of the power spectrum should be revisited first. We compare the GW spectra resulting from $\mathrm{PT}$ occurring at temperatures in the range [100 GeV, $100 \mathrm{PeV}]$ with the sensitivities of LISA, BBO and LIGO correlated third generation (and taken from [26]). The BBO sensitivity is approximate and may change in the final design. We are actually using the sensitivity of BBO Corr, its correlated extension, which correlates two detectors, namely, two LISA-like constellations (each LISA-like constellation orbits around the Sun at $1 \mathrm{AU}$ and consists in three spacecrafts in a triangular configuration) that will allow to do correlations to measure the stochastic background. As discussed in Section II, we take into account as well the irreducible background due to extragalactic white dwarf (WD) binaries.

For each temperature, we are making a full scan of the $\left(\alpha, \beta / H_{*}\right)$ parameter space and determine the regions where at least one of the peaks is observable. According to Eqs. (9)-(12), various situations can arise 2:

(i) For relatively low $\alpha$, the turbulence and collision peaks are well separated and can be observed. This is the ideal situation as the observability of these two peaks would be a smoking gun for the phase transition origin of these GW. The ratio of the two peak frequencies is a predicted function of $\alpha$. In some cases, the turbulence peak is at too low frequency to be observed by LISA or BBO but the minimum separating the two peaks is visible.

(ii) At larger $\alpha(\gtrsim 0.64)$, the collision peak is hidden by the high-frequency tail of the turbulence peak. However, there is a characteristic change of slope in the high-frequency tail. Depending on the temperature of the transition, this change of slope can be observed or not.

Our contour plots show the region where the turbulence peak is observable and the region where either the collision peak or the slope change is visible, at LISA (Fig. 3), BBO (Fig. 4) and LIGO (Fig. 5). The vertical line separates the low $\alpha$ region where the two peaks are well separated from the large $\alpha$ region where only the change of slope is visible. The lower horizontal bound is due to the fact that we cut the sensitivity of both LISA and BBO at $10^{-4} \mathrm{~Hz}$. Because of this frequency limit in the sensitivity, we cannot probe phase transitions below a $\sim \mathrm{GeV}$ and thus the QCD phase transition. In the BBO plots, we show the very important effect of the WD foreground on the detectability at BBO.

Note that we could have also made contours corresponding to cases where none of the peaks are observable but the high or low frequency tails can still be detected. This will clearly enlarge the detectability region and this is work in progress.

\section{A. $T=100 \mathrm{GeV}$}

LISA will be able to detect the peak of GW from a $100 \mathrm{GeV}$ first-order PT only if it is extremely strong $(\alpha \gtrsim$ 0.5 or $\beta / H \lesssim 1000$ ). The Higgs potential of the MSSM does not satisfy this requirement but it can in the NMSSN [13]. Higgs potentials with negative quartic couplings can also trigger strong EWPT as was shown in [24]. The corresponding prospects for $\mathrm{GW}$ detection will be presented in details elsewhere [25]. There are also exciting large signals expected from the high temperature behavior of a warped extra dimension [22]. If $T$ is $\sim 500 \mathrm{GeV}$ rather than $100 \mathrm{GeV}$, the GW peak will coincide with LISA's best sensitivity frequency and a larger region will be detectable. The prospects for detection of GW from the $100 \mathrm{GeV}$ EWPT are very good at BBO. At these low frequencies, the WD foreground is lower. For instance, the turbulence peak for $\alpha \gtrsim 0.3, \beta / H \sim 200$, is above the WD foreground and can be seen by BBO. Note also that at $T=$ $500 \mathrm{GeV}$, values of $\beta / H$ as large as $10^{4}-10^{5}$ can be probed.

\section{B. $T=1 \mathrm{TeV}$}

It is quite exciting that a $1 \mathrm{TeV}$ PT with $\alpha \geq 0.4, \beta / H \sim$ 200 can be seen by LISA. Recent radical proposals to address the hierarchy problem predict rich new phenomena 

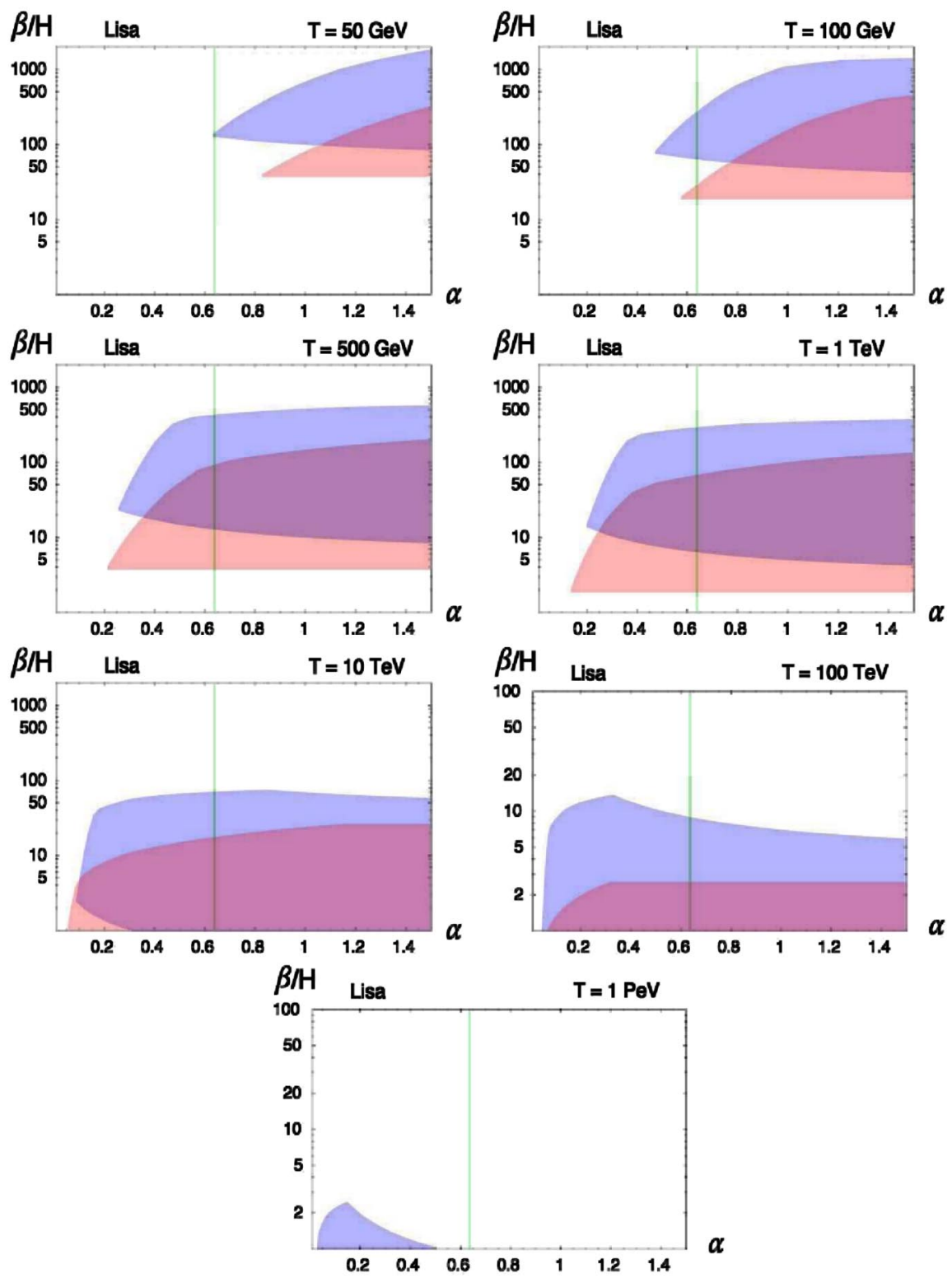

FIG. 3 (color online). Contours delimiting the region in the $(\alpha, \beta / H)$ plane for which there is an observable peak at LISA. The upper blue region is for the turbulence peak while the lower red one is the region where either the collision peak or the point of slope change is visible. Left of the vertical green line, the collision peak is visible.

at the $\mathrm{TeV}$ scale. For instance, new dimensions at a $\mathrm{TeV}$ could give rise to observable signals at LISA [22]. There was also a recent study of the high temperature behaviors of little Higgs theories where it was shown that EW symmetry was restored precisely at a temperature of order $\sim 1 \mathrm{TeV}$ [27]. This transition appeared to be first-order. A more detailed analysis would be required to determine whether this PT could be strong enough to lead to an observable spectrum of GW at LISA. Unfortunately, this takes place in the regime where the effective theory ceases to be under control. This is nevertheless an interesting prospect.

If $\beta / H \geqslant 100$, the turbulence peak is above the WD foreground for $\alpha \geqslant 0.2$, and thus can be seen by BBO. In addition, BBO can see the high-frequency tail of the collision peak which covers good part of, if not entirely, the inflation signal.

\section{C. $T=10 \mathrm{TeV}$}

At these temperatures, the peak cannot be probed by LISA, unless $\beta / H<10^{2}$. On the other hand, LISA can still probe the low-frequency tail of these spectra and is therefore a compelling tool to probe scales that LHC will not be able to reach. As the temperature increases, the peaks are shifted to higher frequencies, thus the effect of the WD foreground becomes less significant and quite weak first-order phase transitions can be probed. And the 

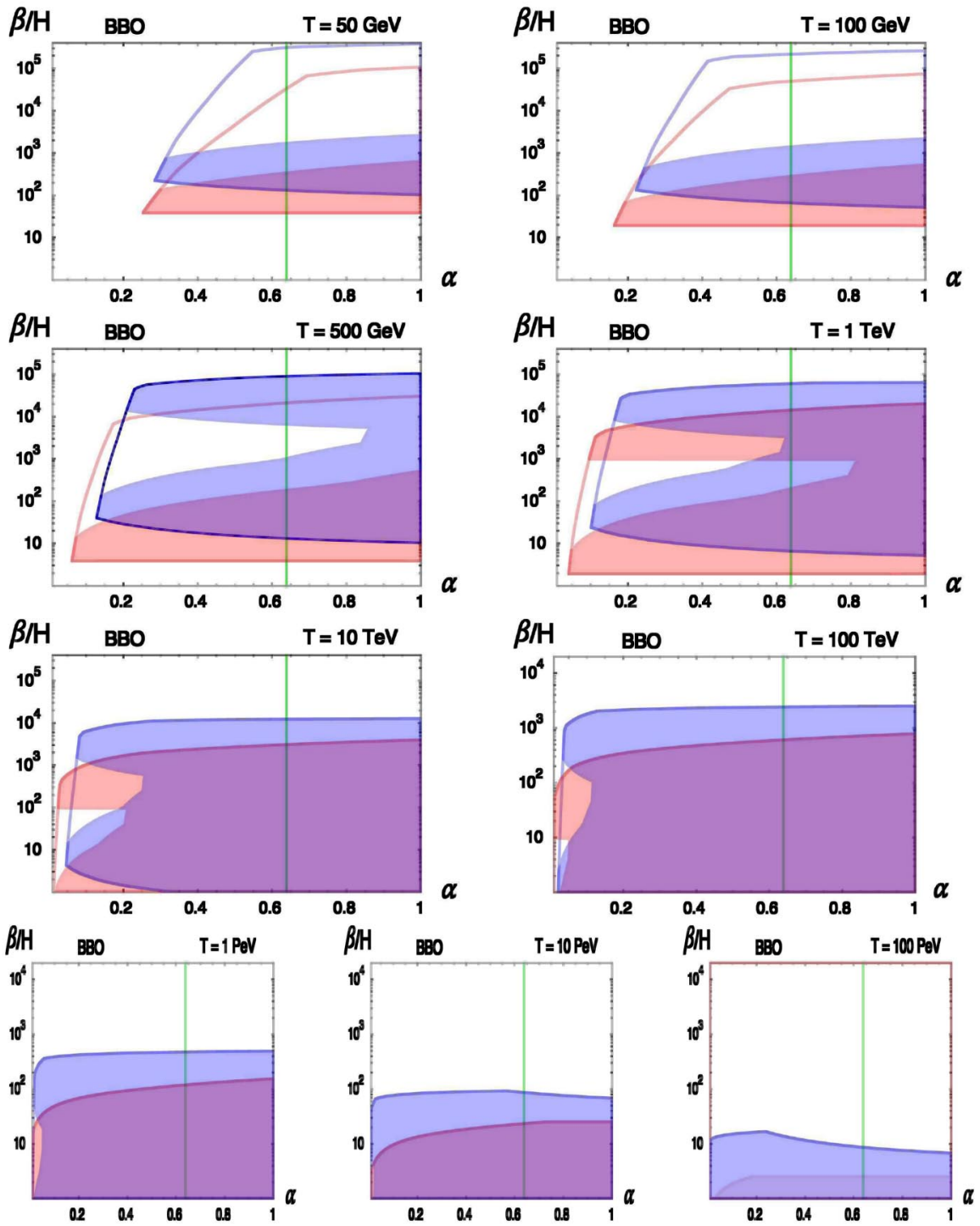

FIG. 4 (color online). Same as Fig. 3 but for BBO. The effect of including the constraint from the irreducible WD foreground is displayed and limits the observable regions from the uncolored ones to the ones in plain colors. As the temperature increases, the peaks are shifted to higher frequencies, thus the effect of the WD foreground becomes less significant.

high-frequency tail of the collision peak can entirely screen the inflation signal, depending on the scale of inflation (see Fig. 6). If $\beta / H \sim 1000$, it is possible to see both the turbulence and the collision peaks for $0.1 \lesssim \alpha \lesssim 0.64$ and assuming that the inflationary scale is sufficiently low. For $\beta / H \sim 200$, the collision peak can be seen for
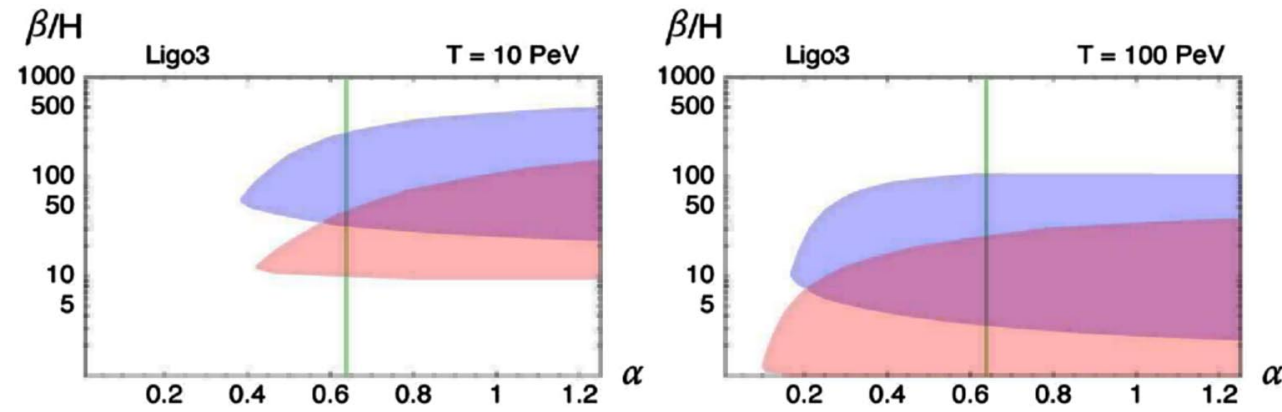

FIG. 5 (color online). Same as Fig. 3 but for LIGO-III. 


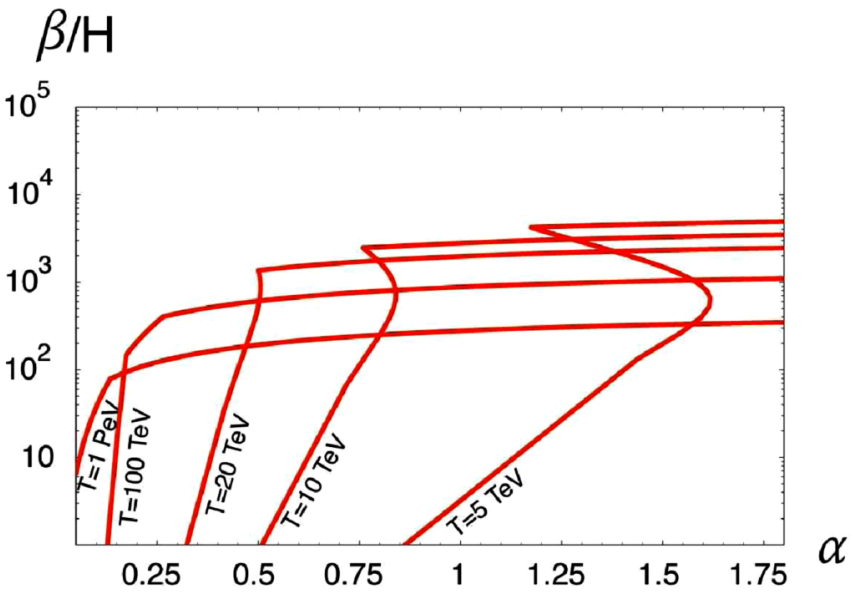

FIG. 6 (color online). Below each line (each one associated with the temperature of the phase transition), the gravitational wave signal at BBO from 1st order phase transitions entirely masks the signal expected from inflation. This plot strongly depends on the scale of inflation, which was chosen here to be $E_{I}=3.4 \times 10^{16} \mathrm{GeV}$.

$\alpha$ as low as $\sim 0.05$ if the inflationary signal is below the BBO sensitivity.

\section{D. $T=100 \mathrm{TeV}$}

If $\beta / H \sim 200$ and $\alpha \geqslant 0.2$ the inflation signal is for sure entirely covered. If the inflation scale is below $\sim 5 \times$ $10^{15} \mathrm{GeV}, \alpha$ as low as 0.05 could be detected at BBO. Two peaks can be seen if $0.1 \lesssim \alpha \lesssim 0.64$ and $\beta / H \sim 200$. At larger $\beta / H$ only the turbulence peak will be seen.

\section{E. $T=10^{7} \mathrm{GeV}$}

This is a particularly interesting case as the same signal could be observed by both BBO and Ligo-III. Specifically, a phase transition with $\alpha=0.8$ and $\beta / H \sim 200$ would give a turbulence peak observable by Ligo-III while the low frequency tail would be observable by BBO. In this example, the inflation signal would be hidden except in a very narrow frequency range between $50 \mathrm{mHz}$ and $80 \mathrm{mHz}$.

Interesting signatures end at this energy scale. Phase transitions at $T \geqslant 10^{8} \mathrm{GeV}$ cannot be probed by any of the planned interferometers.

\section{CONCLUSION}

We have shown that the GW background from early universe phase transitions may become relevant for a second generation detector such as the $\mathrm{BBO}$, which is so far motivated to detect the $\mathrm{GW}$ background produced during inflation. LISA, LIGO and BBO will be able to probe part of the history of the universe in the temperature range
$100 \mathrm{GeV}-10^{7} \mathrm{GeV}$. The $\mathrm{GW}$ signal coming from particle physics phase transitions is directly related to the scalar potential describing the evolution of the order parameter. Observation or nonobservation of GW will allow to put constraints on the parameters of these potentials. The measurement of the GW spectrum (peak frequency and intensity) can discriminate among different models (once combined with experimental measurement at colliders, for instance, once knowing the Higgs mass) and put constraints on the model parameters. For example, at LHC, we will be able to measure the Higgs mass but not the quartic or cubic self coupling of the Higgs. Only a linear collider can provide this information, which timescale could be beyond LISA. LISA could start constraining model parameters before a linear collider. In addition, LISA is sensitive to the $10 \mathrm{TeV}$ scale which is beyond the reach of the near future collider experiments.

The gravitational wave signal from phase transitions at around $10-100 \mathrm{TeV}$ temperatures could entirely screen the signal from inflation, which detection is one of the main motivations for building BBO.

We emphasize that our quantitative analysis can only be indicative given the uncertainties both at the experimental and theoretical level. The sensitivities of LISA, LIGO-III and $\mathrm{BBO}$ will certainly change during the next years. On the theoretical side, we use the estimate of the GW power spectrum of $[6-9,11,12,14]$ which is enough for the point of this paper. However, it certainly deserves improvement. We do not venture into this aspect in this work and just encourage that this question be reexamined given the exciting experimental prospects for GW detection from phase transitions we demonstrated in this analysis.

\section{ACKNOWLEDGMENTS}

We are indebted to Alessandra Buonanno for very useful discussions. We also thank A. Nicolis for a clarification on his work, M. Maggiore for pointing out some reference as well as C. Caprini and R. Durrer for discussions and finally James Wells for stimulating conversations at the early stage of this project.

Note added-As this work was being completed, Ref. [19] appeared where they re-examined the calculation of the gravitational wave background from turbulence. They disagree with and correct the dispersion relation used for gravitational waves in $[11,12,14]$. This leads, in particular, to a different prediction for the peak frequency as well as a different spectral dependence. Re-examination of the bubble collision spectrum is underway [20]. These new results can marginally affect the detectability regions of Figs. 3-5 but the overall conclusions will remain the same. 
[1] URL http://www.ligo.caltech.edu/.

[2] URL http://www.virgo.infn.it/.

[3] URL http://lisa.jpl.nasa.gov/.

[4] T. L. Smith, M. Kamionkowski, and A. Cooray, Phys. Rev. D 73, 023504 (2006).

[5] URL universe.nasa.gov/program/bbo.html.

[6] A. Kosowsky, M. S. Turner, and R. Watkins, Phys. Rev. Lett. 69, 2026 (1992).

[7] A. Kosowsky, M. S. Turner, and R. Watkins, Phys. Rev. D 45, 4514 (1992).

[8] A. Kosowsky and M. S. Turner, Phys. Rev. D 47, 4372 (1993).

[9] M. Kamionkowski, A. Kosowsky, and M. S. Turner, Phys. Rev. D 49, 2837 (1994).

[10] K. Kajantie, M. Laine, K. Rummukainen, and M.E. Shaposhnikov, Phys. Rev. Lett. 77, 2887 (1996); K. Rummukainen, M. Tsypin, K. Kajantie, M. Laine, and M.E. Shaposhnikov, Nucl. Phys. B532, 283 (1998); F. Csikor, Z. Fodor, and J. Heitger, Phys. Rev. Lett. 82, 21 (1999).

[11] A. Kosowsky, A. Mack, and T. Kahniashvili, Phys. Rev. D 66, 024030 (2002).

[12] A. D. Dolgov, D. Grasso, and A. Nicolis, Phys. Rev. D 66,
103505 (2002).

[13] R. Apreda, M. Maggiore, A. Nicolis, and A. Riotto, Nucl. Phys. B631, 342 (2002).

[14] A. Nicolis, Classical Quantum Gravity 21, L27 (2004).

[15] R. Ballantini et al., gr-qc/0502054.

[16] M. Maggiore, Phys. Rep. 331, 283 (2000).

[17] A. J. Farmer and E. S. Phinney, Mon. Not. R. Astron. Soc. 346, 1197 (2003).

[18] C. Cutler and J. Harms, Phys. Rev. D 73, 042001 (2006).

[19] C. Caprini and R. Durrer, Phys. Rev. D 74, 063521 (2006).

[20] C. Caprini, R. Durrer, and G. Servant (unpublished).

[21] P. J. Steinhardt, Phys. Rev. D 25, 2074 (1982).

[22] L. Randall and G. Servant, hep-ph/0607158.

[23] G. D. Moore, J. High Energy Phys. 03 (2000) 006.

[24] C. Grojean, G. Servant, and J. D. Wells, Phys. Rev. D 71, 036001 (2005).

[25] C. Delaunay, C. Grojean, G. Servant, and J.D. Wells (unpublished).

[26] A. Buonanno, G. Sigl, G. G. Raffelt, H. T. Janka, and E. Muller, Phys. Rev. D 72, 084001 (2005).

[27] J. R. Espinosa, M. Losada, and A. Riotto, Phys. Rev. D 72, 043520 (2005). 